THE EFFECTS OF PROGENITOR MASS LOSS ON THE PROPERTIES OF A SUPERNOVA REMNANT

T.A. Lozinskaya

Sternberg Astronomical Institute, Moscow, USSR

Abstract: Observations of ring nebulae around WR and $0_{f}$ stars, including those in $O B$ associations, have been used to give information on the pre-supernova environment.

The nature and evolution of a supernova remnant (SNR) of any age is strongly dependent on the action of the progenitor on the ambient gas. The main factors modifying the pre-SN environment are:

1 . ionizing radiation, which is probably accompanied by the reactive displacement of photo-evaporated cloudlets (Mckee et al., 1984), 2. stellar winds (both 1. and 2. change in the course of the evolution of the star), and 3. ejection of a slow shell. In earlier work the influence of the progenitor on the ISM has been taken into account by assuming an idealized density distribution in the SN vicinity.

Observations of ring nebulae around WR and $0_{f}$ stars make it possible to study directly the "preparation" of the surrounding interstellar gas by a massive progenitor before the SN explosion. Proceeding from the data of our investigations (Lozinskaya, 1982, 1983, 1986; Bochkarev et al., 1987) we can draw the following conclusions about the ISM parameters around WR and $0_{f}$ stars, which are possible precursors of SNe of type II and of the Cassiopeia-A type.

1. Four types of nebulae excited by WR stars (Chu, 1981) and $0_{f}$ stars (Lozinskaya, 1982) are observed. They are amorphous $\left(R_{a}\right)$ and shell-like $\left(R_{s}\right)$ HII regions, wind-blown bubbles $(W)$ and stellar ejecta $(E)$. Their parameters are listed in the following table.

\begin{tabular}{|c|c|c|c|c|}
\hline Type & $R_{a}$ & $\mathrm{R}_{S}$ & $W$ & $E$ \\
\hline$R(p c)$ & $10-50$ & $10-30$ & $1-10$ & $1-5$ \\
\hline$V_{\exp }(\mathrm{km} / \mathrm{s})$ & $\begin{array}{l}<10 \\
\text { (chaotic) }\end{array}$ & $\leqslant 10$ & $20-100$ & $20-50$ \\
\hline Mass $\left(M_{\mathcal{O}}\right)$ & $10^{2}-10^{3}$ & $100-500$ & $10-100$ & $1-5$ \\
\hline Structure & irregular & $\begin{array}{l}\text { shel 1 } \\
\Delta R / R \sim 0.5\end{array}$ & $\begin{array}{c}\text { filamentary } \\
\text { shell } \\
\Delta R / R \sim 0.1\end{array}$ & $\begin{array}{l}\text { knotty } \\
\text { shell }\end{array}$ \\
\hline
\end{tabular}


The kinematical age of $W$ - and $E$ - shells is found to be about $(1-5)$ $10^{4} \mathrm{yr}$. Soft $X$-ray emission found in NGC 6888 testifies to the fact that, in accordance with the classical theory, wind-blown bubbles are filled by hot plasma. Distinct shell-like structure (i.e. $R_{S}-$, W- or E-type nebulae) is observed in about $40-50 \%$ of HII regions excited by $W R$ and $0_{f}$ stars.

2. Multishell structure is often observed: small E- and W- type shells are surrounded by extended $R_{S}-$ or $R_{a}-$ nebulae. The most impressive example is provided by the system of four concentric shells around the $0_{f}$ star HD 148937: the stellar ejecta NGC 6164-5 (R 1 pC),

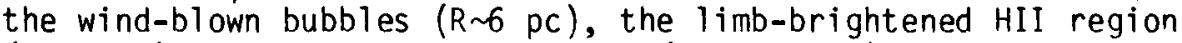
$(R \sim 25 \mathrm{pc})$ and the outer dust shell (R 25-30 pc); see Fig. 1 .

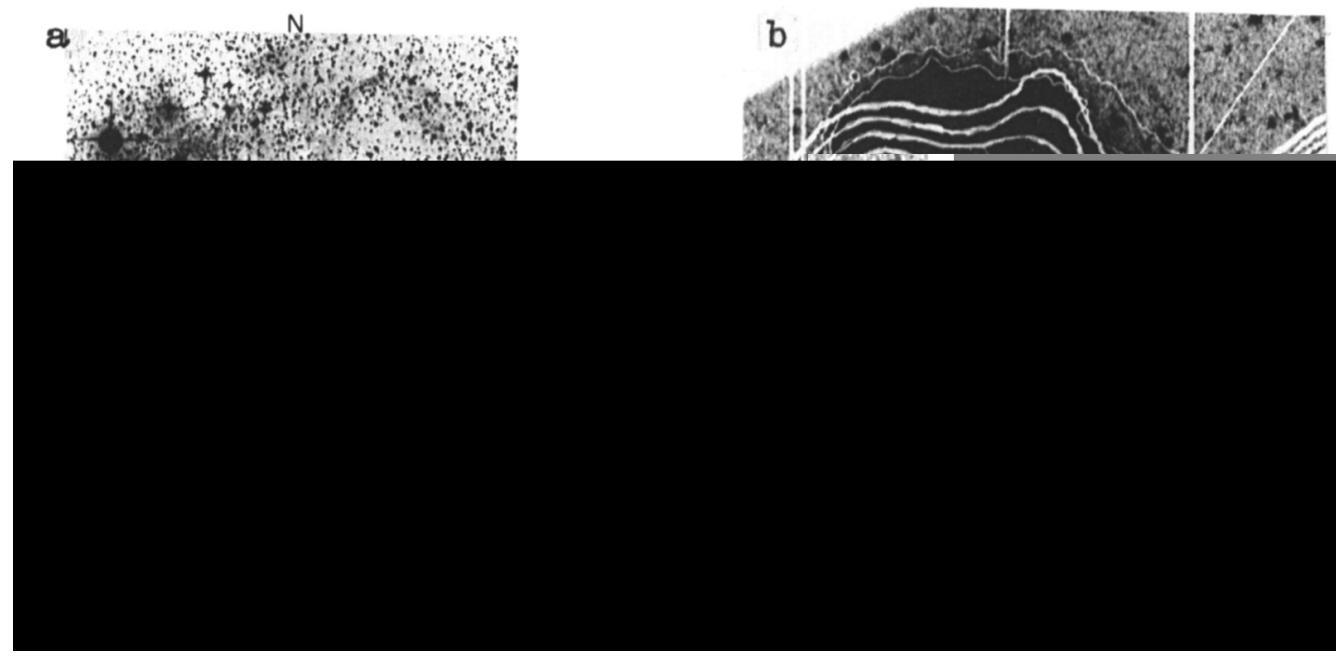

Figure 1: a - NGC 6164-5, two inner shells $E$ and $W$ types; image from Bruhweiler et al., 1981

b - NGC 6888, OIII 5007 A image together with Einstein Observatory $X$-ray contours in the range $0.2-3.5 \mathrm{keV}$

Such a structure shows that mass loss in the form of stellar winds and of slow ejecta occurs in the same star and that both processes are not greatly separated in time. Thus the interstellar gas around a massive SN-precursor is characterized by a complicated multilayer structure with sharp density gradients formed by UV-radiation, stellar winds and slow ejecta. The propagation of the SN-blast wave in such a medium must be accompanied by formation of secondary (forward and reflected) shock waves, see e.g. Itoh and Fabian, 1984. This might manifest itself in the optical and $X$-ray emission of young and middle-aged SNRs. Indeed, in Cassiopeia A we can distinguish the two-component SN ejecta (fast-moving optical knots and the inner X-ray shell) interacting with the two-component matter lost by the progenitor (quasi-stationary flocculi which most likely represent the slow ejecta of a WR star and the outer X-ray shell).

3. Wind-blown bubbles and ejecta are often characterized by an 
elongated form; examples are NGC 6888, 2359, 6164-5 and the second shell in the same system. In E-nebulae such a structure is most probably the result of bipolar ejection. In $W$-nebulae it may be caused by inhomogeneity of the ambient ISM as well as by a biconical stellar wind. In particular, our latest investigations of the nebula NGC 6888 (in preparation) show that the structure and kinematics of the optical filaments may be explained by inhomogeneity of the ISM (see also Johnson and Songsathaporn, 1981). At the same time the X-ray picture of two hot spots on opposite sides of the nebula (see Fig. 1), may be caused by an asymmetrical stellar wind.

Irrespective of the formation mechanism such axially symmetric distribution of the density in the environment of the progenitor may provide an interpretation for the commonly observed "barrel-like" and "biannular" morphology of radio supernova remnants (see Manchester, 1987). The other explanation which has been proposed by Manchester biconical relativistic particle flow from a central pulsar - is hardiy applicable to young shell type SNRs such as SN 1006 because of the absence of a detectable synchrotron nebula. On the other hand, an explanation invoking the asymmetries of swept-up or ejected gas around the pre-SN star can be relevant to all SNRs.

It should be noted in this respect that bipolar flows from pre-planetary stars and bipolar or annular forms of many planetary nebulae indicate that a similar environment is typical also for the low mass progenitors of SN 1 .

For old SNRs of large size the influence of radiation, mass loss and previous SN explosions of nearby stars all become relevant. If a SN has exploded in an OB association (where massive stars, the presumed progenitors of supernovae, occur) these factors seem to determine the pre-SN environment.

Shells and supershells around $O B$ associations are a large subject and I shall not dwell on it at length. I would only like to emphasize

\section{Figure 2:}

Schematic representation of multishell formations around $\mathrm{OB}$ associations.

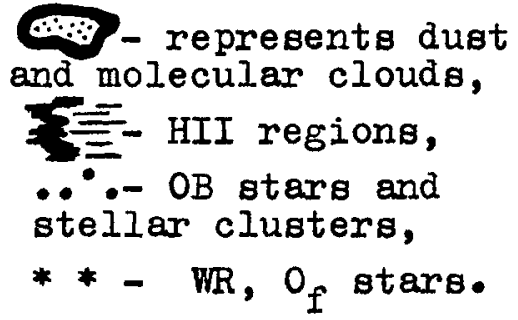

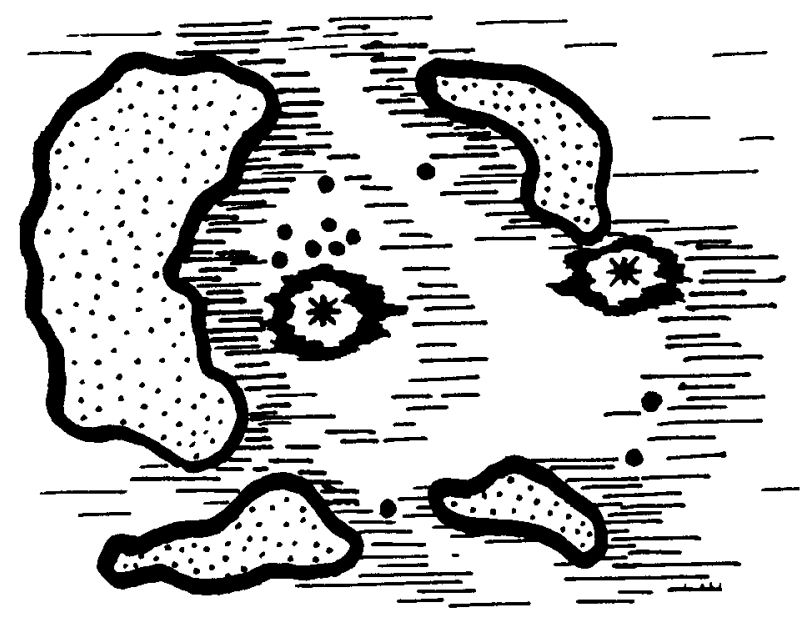


that multishell hierarchical structure is observed here as well in the distribution of emission nebulae, dust and molecular clouds.

Fig. 2 shows the general scheme of such a shell hierarchy which we have detected around the associations Cas 0B2 (Lozinskaya et al., 1986), Cep 0B4 (Lozinskaya et al., 1987) and Cyg OB1 (Lozinskaya and Sitnik, in preparation). Inside the common shells around $O B$ associations there are shell-like formations of smaller size related to young clusters and the smallest ring nebulae around the isolated sources of strong wind: WR and $0_{f}$ stars. Such a multishell structure is observed more easily in the "face-on" LMC, the LMC 2 region being the most distinctive example (see Caulet et al., 1982).

\section{References}

Bochkarev, N.G., Lozinskaya, T.A., Piskunov, N.E., Pravdicova, V.V., Sitnik, T.G., 1987, Proc. of A11-Union Meeting "WR stars and related objects", Tartu, 1986

Bruhweiler, F.C., Gull, T.R., Henize, K.G., Cannon, R.D., 1981, Ap. J., 251,126

Caulet, A., Deharveng, L., Georgelin, Y.M., Georgel in, Y.P., 1982, Astr. Ap. 110,185

Chu, Y.-H., 1981, Ap.J. 249, 197

Itoh, A., Fabian, A.C., 1984, MNRAS 208, 645

Johnson, P.G., Songsathaporn, R., 1981, MNRAS 195, 51

Lozinskaya, T.A., 1982, Astrophys. Space Sci. $\overline{87}, 313$

Lozinskaya, T.A., 1983, Sov. Astron. Lett. - Pisma Astron. J., 9, 469

Lozinskaya, T.A., 1986, "Supernovae and Stellar Winds; Interaction with the Interstellar Gas", Moscow, Nauka, 304 pages

Lozinskaya, T.A., Sitnik, T.G., Lomovskiy, A.I., 1986, Astrophys. Space Sci., 121, 357

Lozinskaya, T.A., Sitnik, T.G., Toropova, M.S., 1987, Sov. Astr. J. Astron. Zh., 64, in press.

Manchester, R.N., 1987, Astr. Ap., 171, 205

Mckee, C.F., Van Buren, D., Lazareff, B., 1984, Ap. J. Lett., 278, L115 